\title{
A multibaker map for shear flow and viscous heating
}

\author{
László Mátyás, ${ }^{(1)}$ Tamás Tél, ${ }^{(1)}$, and Jürgen Vollmer ${ }^{(2,3)}$ \\ (1) Institute for Theoretical Physics, Eötvös University, P. O. Box 32, H-1518 Budapest, Hungary. \\ (2) Fachbereich Physik, Univ.-GH Essen, 45117 Essen, Germany. \\ (3) Max Planck Institute for Polymer Research, Ackermannweg 10, 55128 Mainz, Germany.
}

(November 19, 2018)

\begin{abstract}
A consistent description of shear flow and the accompanied viscous heating as well the associated entropy balance is given in the framework of a deterministic dynamical system. A laminar shear flow is modeled by a Hamiltonian multibaker map which drives velocity and temperature fields. In an appropriate macroscopic limit one recovers the Navier-Stokes and heat conduction equations along with the associated entropy balance. This indicates that results of nonequilibrium thermodynamics can be described by means of an abstract, sufficiently chaotic and mixing dynamics. A thermostating algorithm can also be incorporated into this framework.
\end{abstract}

05.70.Ln, 05.45.Ac, 05.20.-y, 51.20.+d

\section{INTRODUCTION}

Shear flows provide one of the paradigms of transport processes [1] 5]. The importance of chaos in the equations underlying macroscopic shearing has recently been addressed by various numerical studies [6 9], which to some extent were supported by kinetic theory [3,9] and rigorous mathematical work [7,10]. In contrast, however, a simple, exactly solvable model based on a lowdimensional chaotic dynamics - whose mixing property would be the cause of irreversibility - has not yet been established. For material and heat transport such type of models have helped to understand the physical content of thermostating schemes used in numerical simulations 11-14 (cf. however 15] for open questions). In the present article we introduce a similar model for shear flows in the hope that it can also serve such a purpose. The approach will be based on multibaker maps. Previous works in this spirit successfully described the phenomena of diffusion 16 18], conduction in an external field 19 22], chemical reactions 23], thermal conduction [24], and cross effects due to the simultaneous presence of an external field and heat conduction 25, 26].

Our aim is to model a sheared fluid confined between two parallel walls at the coordinates $x=0$ and $x=L$ (Fig. (1). The flow is assumed to be two-dimensional in the $(x, y)$ plane. Shear is induced by prescribing different $y$ components of the average velocities $\mathbf{v}$ of particles close to the respective walls. In order to make the calculations more transparent, we confine the discussion to cases where the driving is sufficiently weak to induce only a laminar flow, i.e., to cases where the velocity of particles is always directed in the vertical direction such that $\mathbf{v}=(0, v(x))$. For this system we establish a local entropy balance that covers time dependent effects and does not rely on the implementation of boundary conditions.

Three different boundary conditions for dealing with the dissipated heat are considered: (i) In the simplest case the system is isolated. A stationary linear velocity profile emerges in that case, and the temperature becomes uniform. No steady state is reached, however, due to a constant increase of temperature in response to the viscous heating. In addition, we consider systems where (ii) there is a bulk thermostat uniformly taking out the viscous heat, and (iii) the temperature is fixed to the same value at both boundaries so that the asymptotic temperature profile is stationary, but no longer uniform.

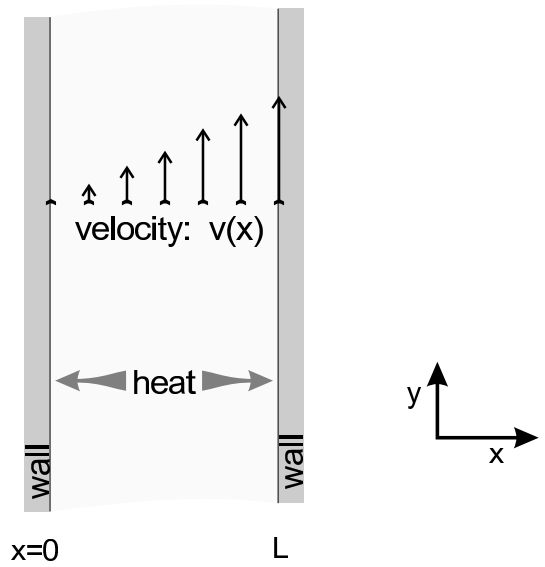

FIG. 1. Graphical illustration of the shear flow. The system is confined between two walls at positions $x=0$ and $L$. The walls move relative to each other in the $y$ direction, thus inducing a velocity profile $v(x)$ indicated by vertical arrows. For a fixed temperature at the walls this leads to an accompanying heat flow in the $x$ direction (gray arrows).

The paper is organized as follows: In Sect. II we recall basic notions of irreversible thermodynamics that are to be recovered in a suitable continuum limit of the multibaker dynamics. In Sect. III we introduce the model, and establish the evolution equations for the velocity and the temperature field. This allows us to address the entropy dynamics and its balance equation (Sect. IV]). Subsequently, in Sect. V the macroscopic limit of the resulting equations is worked out. The global behavior at differ- 
ent boundary conditions is compared to irreversible thermodynamics in Sect. VII, and conclusions are drawn in Sect. VIII.

\section{IRREVERSIBLE THERMODYNAMICS}

In this section we recall the thermodynamic description of shear flows accompanied by viscous heating. The picture is simplified by considering an incompressible fluid at constant pressure.

\section{A. Transport equations}

For a system with constant density and pressure the temperature $T$ is the only relevant state variable, and for a complete description one also has to specify the velocity field $\mathbf{v}$ of the fluid [1,2]. The thermodynamic state variables are the velocity field $\mathbf{v}$ and the temperature $T$ [2]. Mass conservation is expressed by a continuity equation. For incompressible fluids the uniform mass density $\rho$ implies that the flow is divergence free, i.e.,

$$
\partial_{i} v_{i}=0
$$

Here $i=x, y$ labels the components of the local flow velocity $\mathbf{v} \equiv\left(v_{x}, v_{y}\right)$, and we adopted the Einstein convention, i.e., summation over repeated indices. The equation of motion for the velocity components is given by the Navier-Stokes equation. For the case of a negligible pressure gradient it reads

$$
\frac{d}{d t} v_{i}=\nu \partial_{j} \partial_{j} v_{i}
$$

where $\nu$ is the kinematic viscosity, and $d / d t$ is the total time derivative.

The system of equations is closed [2] by the equation

$$
\frac{d}{d t} T=\frac{\lambda}{\rho c_{V}} \partial_{i} \partial_{i} T+\frac{1}{2} \frac{\nu}{c_{V}}\left(\partial_{k} v_{l}+\partial_{l} v_{k}\right)\left(\partial_{k} v_{l}+\partial_{l} v_{k}\right)
$$

for the temperature evolution. Here, $c_{V}$ is the specific heat at constant volume, and $\lambda$ is the thermal conductivity.

\section{B. Entropy balance}

The balance equation of the entropy density $s$ is

$$
\begin{aligned}
\partial_{t} s & =\sigma^{(i r r)}+\Phi, \\
\Phi & =-\partial_{i} j_{i}^{(s)}+\Phi^{(t h)},
\end{aligned}
$$

where $\sigma^{(i r r)}$ is the irreversible entropy production reflecting the viscous heating of the flow, $\Phi$ the entropy flux, and $\mathbf{j}^{(s)}$ is the entropy-current density. The term $\Phi^{(t h)}$ models an entropy flux let into a heat bath. In the bulk of a hydrodynamic system this term typically vanishes, but it takes non-vanishing values wherever there is a flux into the environment (i.e., for instance at the boundaries when there is a heat flux through the walls). In simulations where one does not desire to focus on the effects of temperature gradients, and applies a thermostating algorithm, a non-vanishing $\Phi^{(t h)}$ can appear even in the bulk of the system. We say that a system is subjected to an ideal thermostat, when the dissipated heat is directly let into the heat bath. In that case the entropy current vanishes in the steady state, $j_{i}^{(s)}=0$, while $\Phi^{(t h)}$ is nonzero in the bulk, and counterbalances the steady-state entropy production.

For an incompressible fluid in local equilibrium the Gibbs relation $\mathrm{d} s=\mathrm{d} u / T$ applies locally. The evolution equations can be used to evaluate the terms in Eq. (A). A straightforward calculation yields for the rate of irreversible entropy production (cf. for instance 迆, Ch. XII. (23)])

$$
\sigma^{(i r r)}=\lambda \frac{\left(\partial_{i} T\right)\left(\partial_{i} T\right)}{T^{2}}+\frac{\nu \rho}{2 T}\left(\partial_{j} v_{k}+\partial_{k} v_{j}\right)\left(\partial_{j} v_{k}+\partial_{k} v_{j}\right)
$$

The associated entropy current takes the form [1, Ch. XII. (22), (24)]

$$
j_{i}^{(s)}=-\lambda \frac{\partial_{i} T}{T} .
$$

It depends only on the local temperature and its gradient. The flow velocity $\mathbf{v}$ does not enter.

\section{Laminar Flow}

For a laminar flow driven by prescribed non-trivial $y$ components of the velocity at the two walls, the velocity field at any position $(x, y)$ takes the form $\mathbf{v} \equiv(0, v(x))$ (see Fig. 1). The $x$ component of the velocity vanishes, and the profile is translational invariant in the $y$ direction (parallel to the walls). We restrict our investigation to cases where the same holds for the temperature such that $T=T(x)$. Consequently, for the laminar flow the transport equations take the form

$$
\begin{aligned}
\partial_{t} v & =\nu \partial_{x}^{2} v, \\
\partial_{t} T & =\frac{\lambda}{\rho c_{V}} \partial_{x}^{2} T+\frac{\nu}{c_{V}}\left(\partial_{x} v\right)^{2},
\end{aligned}
$$

while the rate of irreversible entropy production $\sigma^{(i r r)}$ and the entropy current $j^{(s)}$ can be written as

$$
\begin{aligned}
\sigma^{(i r r)} & =\lambda\left(\frac{\partial_{x} T}{T}\right)^{2}+\frac{\nu \rho}{T}\left(\partial_{x} v\right)^{2}, \\
j^{(s)} & =-\lambda \frac{\partial_{x} T}{T} .
\end{aligned}
$$


The scalar current $j^{(s)}$ denotes the $x$ component of the entropy current, and an analogous convention is adopted for all currents. The $y$ components of the currents vanish in the considered setting.
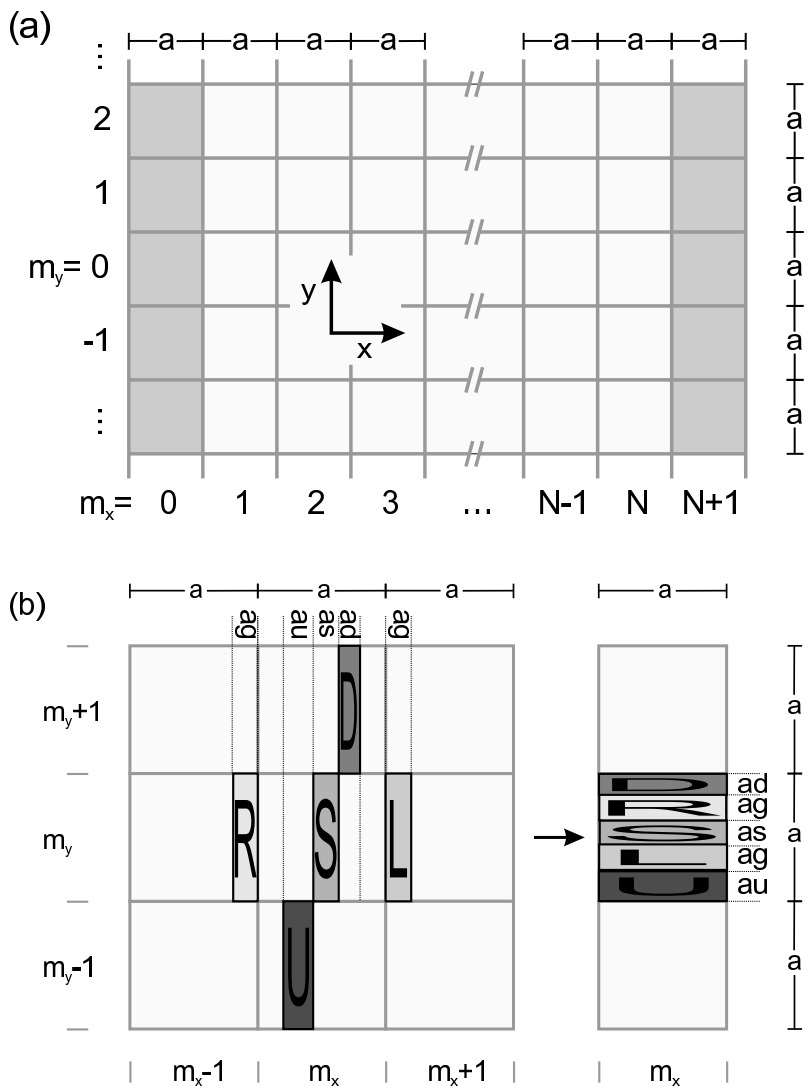

FIG. 2. Graphical illustration of the action of the multibaker map on its phase space $(x, y)$. The indices labeling the columns and rows are given on the lower and left margin, respectively, while their width is indicated at the upper and right margins. (a) The mapping is defined on a domain of $N+2$ identical columns of square cells of size $a \times a$ that are labeled by the indices $m_{x}=0, \cdots, N+1$ and $m_{y}=-\infty, \cdots, \infty$. Boundary conditions on the flow are implemented in the shaded cells $m_{x}=0$ and $N+1$. (b) The action of the map on regions that are mapped into cell $\left(m_{x}, m_{y}\right)$. The contraction and expansion for these regions is indicated by the deformation of the tags $(R, U, S, D, L)$ in the different branches of the map.

\section{THE MULTIBAKER MAP}

In order to model the laminar flow we divide the plane into square cells of size $a \times a$ that are large enough, on the one hand, to admit thermodynamically meaningful averages, but, on the other hand, sufficiently small to neglect gradients across cells. The cells are considered as the regions used in irreversible thermodynamics to define local equilibrium variables. The system shown in Fig. 1 is represented by a rectangular array of $N \equiv L / a$ cells in horizontal direction, and an infinite number of cells vertically (Fig. 2a). Cells are labeled by the indices $m_{x}=0, \cdots, N+1$, and $m_{y}=-\infty, \cdots, \infty$. All cells have the same dynamics except the outermost ones where it is modified to implement boundary conditions.

\section{A. Action of the mapping in the $(x, y)$ plane}

After each time unit $\tau$ every cell is divided into five columns (Fig. 2 2b). The rightmost (R) and leftmost (L) column have width $a g$. They are mapped onto a strip of height $a g$ in cell $\left(m_{x}+1, m_{y}\right)$ and cell $\left(m_{x}-1, m_{y}\right)$, respectively. The left column (U) of widths $a u$ is mapped upwards onto a strip of height $a u$ in cell $\left(m_{x}, m_{y}+1\right)$, and the right one labeled by (D) downwards into $\left(m_{x}, m_{y}-1\right)$, respectively. Region $S$ stays within the cell. In all cases the area of the strips is preserved.

This dynamics is driving two fields. Denoting the composite index $\left(m_{x}, m_{y}\right)$ as $\mathbf{m}$, these fields are

(i) the velocity field $v_{\mathbf{m}}$ describing the mean flow velocity $v(x, y)$ in cell $\mathbf{m}$

(ii) an energy field $w_{\mathbf{m}}$ that represents the kinetic energy of the fluid in cell $\mathbf{m}$.

The dynamics on this two-dimensional lattice can be considered as a model of the velocity and energy transport in the configuration space. In order to obtain a faithful representation of the entropy balance, however, one has to consider the phase-space dynamics. To define the dynamics in the analog of a $\mu$-space, we take into account the translation invariance of the problem. The velocity and energy fields can take on different values in the columns $m_{x}=0, \cdots, N+1$ of Fig. 2a, but the fields have to be uniform within every column. In this respect it is not necessary to follow the dynamics in the $y$ direction, and one achieves a quasi one-dimensional dynamics in an $(x, p)$ space, where $p$ represents the non-trivial momentum-like variable of the model.

\section{B. Action of the mapping in the $(x, p)$ space}

The domain of the multibaker in the $(x, p)$ space is shown in Fig. Ba. It comprises a chain of $N+2$ cells of size $a \times b$, that for sake of more condensed notations are labeled by the index $m$. The middle $N$ cells represent the bulk, and two additional ones are used to implement boundary conditions (cf. Section $\mathrm{VI}$ ). The parameter $b$ sets the momentum scale. It will not play any role in thermodynamic considerations.

In order to maintain the same dynamics in the transport direction in the two representations of the flow, each cell is divided into three columns of size $a g, a \hat{s}$ and $a g$, where $g$ is the same as above and $\hat{s}=1-2 g$. The left and right columns of cell $m$ are mapped into a strip of height $a g$ in cell $m+1$ and cell $m-1$, respectively, as shown in Fig. $3 \mathrm{~b}$. The middle column of size $a \hat{s}$ is squeezed and 
stretched onto a strip of height $a \hat{s}$ and remains in the same cell.

This multibaker dynamics drives the velocity and energy fields. Their values might depend on the phasespace coordinate. Hence, we are dealing with the bivariate distribution of the velocity $v(x, p)$ and kinetic energy $w(x, p)$ within each cell $m$. Only the cell averages, $v_{m}$ and $w_{m}$ appear in the transport equations. The dependence of $v(x, p)$ and $w(x, p)$ on the phase-space coordinates contributes essentially to the entropy dynamics, however.

(a)

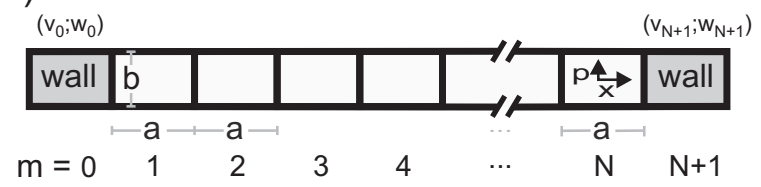

(b)

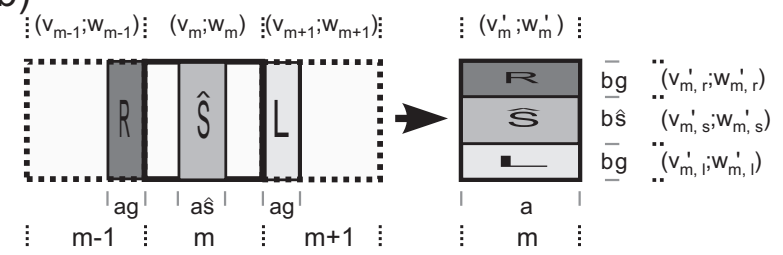

FIG. 3. Graphical illustration of the $(x, p)$ multibaker representation of the laminar flow. (a) Domain of the map. The outermost cells (indicated as grey boxes) are again used to implement boundary conditions. (b) Action in the bulk. The average values of the fields $v(x, y)$ and $w(x, y)$ on the cells [cf. Eqs. (12) and (18)] and strips [cf. Eqs. (11) and (19)] are given on the margins.

\section{The dynamics of the velocity field}

The mass density $\rho_{m}$ is proportional to the average phase-space density $\varrho_{m}$, in cell $m$, i.e.,

$$
\rho_{m}=M b \varrho_{m},
$$

where $M$ is a constant of dimension mass. When interpreting the mass density or associated quantities from the point of view of the two-dimensional flow, $\rho_{m}$ is to be understood as a mass density in the $x$ coordinate per unit length in $y$ direction. Hence, $\rho_{m} v_{m}$ represents the preserved momentum density (per unit length) of the hydrodynamic flow. Its evolution expresses momentum conservation. As a consequence, the updated values for the velocity on the strips $(L, \hat{S}, R)$ after one time unit are (cf. the right side of Fig. $3 \mathrm{~b}$ )

$$
\begin{aligned}
& \rho_{m}^{\prime} v_{m, l}^{\prime}=\rho_{m+1} v_{m+1}, \\
& \rho_{m}^{\prime} v_{m, s}^{\prime}=\rho_{m} v_{m}, \\
& \rho_{m}^{\prime} v_{m, r}^{\prime}=\rho_{m-1} v_{m-1},
\end{aligned}
$$

where, $v_{m, l}^{\prime}, v_{m, s}^{\prime}$, and $v_{m, r}^{\prime}$ stand for the velocities of the flow in the regions $(L, \hat{S}, R)$ of cell $m$, respectively. The prime indicates that the updated values of the field are considered. The average momentum $\rho_{m} v_{m}$ of the full cell is the average of the contributions on the different strips, i.e.,

$$
\rho_{m} v_{m}=\left(g \rho_{m, r} v_{m, r}+\hat{s} \rho_{m, s} v_{m, s}+g \rho_{m, l} v_{m, l}\right)
$$

at any instant of time. If the velocity is initially uniform in the full cell (i.e., the values of $v$ in all strips agree), then due to Eqs. (12) and (11) the average velocity of cell $m$ becomes after one time step

$$
\rho_{m}^{\prime} v_{m}^{\prime}=(1-2 g) \rho_{m} v_{m}+g \rho_{m-1} v_{m-1}+g \rho_{m+1} v_{m+1}
$$

Observing that the mass density $\rho$ is uniform and constant in time, the updated value of the cell velocity $v_{m}$ is found to be

$$
v_{m}^{\prime}=v_{m}+g\left(v_{m-1}+v_{m+1}-2 v_{m}\right) .
$$

This evolution can be written in the form of a discrete balance equation

$$
\frac{v_{m}^{\prime}-v_{m}}{\tau}=-\frac{j_{m+1}^{(v)}-j_{m}^{(v)}}{a}
$$

with the discrete current

$$
j_{m}^{(v)}=-\frac{a^{2} g}{\tau} \frac{v_{m}-v_{m-1}}{a} .
$$

In the boundary columns $m=0$ and $N+1$ the dynamics will be augmented by force terms (Sect. VI) in order to fix the velocity to the constant values $v_{0} \equiv v_{L}$ and $v_{N+1} \equiv v_{R}$, respectively, irrespective of the momentum flowing into these cells.

\section{The dynamics of the energy field}

The energy of cell $m$ is obtained by integrating $e_{m} \varrho_{m}$ over the volume $a b$ of the cell. At any instant of time it is the spatial average of the energies $e_{m, l}, e_{m, s}$ and $e_{m, r}$ of the different strips,

$$
e_{m}=g e_{m, l}+\hat{s} e_{m, s}+g e_{m, r}
$$

The difference between the energy $e$ and the translational specific kinetic energy $M v^{2} / 2$ of the flow defines the specific kinetic-energy density $w \equiv e-M v^{2} / 2$, whose macroscopic limit will be proportional to the local temperature. Therefore, on the strip $i=l, s, r$ in cell $m$ one observes kinetic-energy densities $w_{m, i}$ that fulfill

$$
e_{m, i}=\frac{M}{2} v_{m, i}^{2}+w_{m, i},
$$

while the coarse-grained kinetic energy $e_{m}$ obeys at the same time

$$
e_{m}=\frac{M}{2} v_{m}^{2}+w_{m}
$$

Using these definitions and the averaging rule (17) for the energy one finds 


$$
w_{m}=g w_{m, l}+\hat{s} w_{m, s}+g w_{m, r}+\frac{M}{2}\left[g v_{m, l}^{2}+\hat{s} v_{m, s}^{2}+g v_{m, r}^{2}-\left(g v_{m, l}+\hat{s} v_{m, s}+g v_{m, r}\right)^{2}\right]
$$

This shows that the average kinetic energy $w_{m}$ is not the straightforward spatial average of the values $w_{m, i}$ on the strips. Rather intra-cell variations of the velocity field (i.e., a non-trivial distribution of the $v_{m, i}$ ) also contribute to $w_{m}$.

For a thermally closed system the specific kinetic energy $w$ is advected by the flow. The values on the strips after one time unit are then

$$
\begin{aligned}
w_{m, l}^{\prime} & =w_{m+1}, \\
w_{m, s}^{\prime} & =w_{m}, \\
w_{m, r}^{\prime} & =w_{m-1} .
\end{aligned}
$$

From this and the update (11) of the velocities the updated kinetic energy is found to be

$$
\begin{aligned}
w_{m}^{\prime} & =w_{m}+g\left(w_{m-1}+w_{m+1}-2 w_{m}\right)+ \\
& +\frac{g M}{2}\left[\left(v_{m-1}-v_{m}\right)^{2}+\left(v_{m+1}-v_{m}\right)^{2}\right] \\
& -\frac{M}{2}\left[a^{2} g \frac{\left(v_{m-1}+v_{m+1}-2 v_{m}\right)}{a^{2}}\right]^{2} .
\end{aligned}
$$

The terms proportional to $M$ describe the effect of viscous heating in this discrete setting.

In order to model the action of a thermostat, that leads to local changes of the specific kinetic energy due to a heat flux into the environment, an additional thermostat heat source $q_{m}$ is incorporated into the update of $w_{m}$ by multiplying the right-hand side of Eq. (20) by a factor $\left[1+\tau q_{m}\right]$

$$
\begin{aligned}
w_{m}^{\prime} & =\left\{w_{m}+g\left(w_{m-1}+w_{m+1}-2 w_{m}\right)+\right. \\
& +\frac{g M}{2}\left[\left(v_{m-1}-v_{m}\right)^{2}+\left(v_{m+1}-v_{m}\right)^{2}\right] \\
& \left.-\frac{M}{2} g^{2}\left[v_{m-1}+v_{m+1}-2 v_{m}\right]^{2}\right\}\left[1+\tau q_{m}\right] .
\end{aligned}
$$

This equation can be rewritten as

$$
\frac{w_{m}^{\prime}-w_{m}}{\tau}=Q_{m}+\frac{a^{2} g}{\tau} \frac{w_{m+1}-2 w_{m}+w_{m-1}}{a^{2}}
$$

with the full heat source

$$
\begin{aligned}
Q_{m} & =\frac{q_{m}}{1+\tau q_{m}} w_{m}^{\prime} \\
& +\frac{a^{2} g}{\tau} \frac{M}{2}\left[\frac{\left(v_{m-1}-v_{m}\right)^{2}}{a^{2}}+\frac{\left(v_{m+1}-v_{m}\right)^{2}}{a^{2}}\right] \\
& -\frac{\tau}{2} M\left[\frac{a^{2} g}{\tau} \frac{\left(v_{m-1}+v_{m+1}-2 v_{m}\right)}{a^{2}}\right]^{2}
\end{aligned}
$$

The equation for the update of $w$ can be rearranged into a balance equation for the heat per unit volume

$$
\rho \frac{w_{m}^{\prime}-w_{m}}{\tau}=\rho Q_{m}-\frac{j_{m+1}^{(w)}-j_{m}^{(w)}}{a}
$$

which comprises the divergence of the discrete "heat" current

$$
j_{m}^{(w)}=-\frac{a^{2} g}{\tau} \frac{\rho}{M} \frac{w_{m}-w_{m-1}}{a} .
$$

The first contribution to $Q_{m}$ in (23) reflects the action of the thermostat, and the latter two the effect of viscous heating of the fluid. A steady state with a uniform $w$ profile can be found for $Q=0$, which thus mimics an ideal thermostat. In contrast, in a bulk system in the sense of conventional irreversible thermodynamics $q_{m}=0$, and $Q_{m}$ only vanishes when the discrete velocity gradients $v_{m+1}-v_{m}$ and $v_{m}-v_{m-1}$ across the cell vanish.

\section{ENTROPIES AND THEIR TIME EVOLUTION}

\section{A. The coarse-grained and the Gibbs entropy}

The Gibbs entropy for cell $m$ of a multibaker system with a density field $\varrho(x, p)$ and specific kinetic-energy field $w(x, p)$ takes the form 25]

$$
S_{m}^{(G)}=-k_{B} \int_{\text {cell } m} d x d p \varrho(x, p) \ln \left[\frac{\varrho(x, p)}{\varrho^{*}} w(x, p)^{-\gamma}\right]
$$

where $\gamma$ is a free exponent. Since the phase-space density $\varrho(x, p)$ is constant, one can identify the reference density $\varrho^{*}$ with $\varrho(x, p)$, and obtains

$$
S_{m}^{(G)}=k_{B} \gamma \frac{a \rho}{M} \int_{\text {cell } m} \frac{d x}{a} \frac{d p}{b} \ln w(x, p) .
$$

Here, we have already replaced the phase-space density by the mass density (10).

The coarse-grained entropy of cell $m$ is based on the cell-averaged coarse-grained value $w_{m}$ of the kinetic energy $w(x, p)$. For the form (27) of the Gibbs entropy, one obtains

$$
S_{m}=k_{B} a \gamma \frac{\rho}{M} \ln w_{m}
$$

as the coarse-grained entropy.

In order to discuss the thermodynamic time evolution of entropies, one conveniently starts with uniform densities in every cell [21,14. In that case the coarse-grained and the Gibbs entropy initially coincide. After one time step, however, they typically differ. The Gibbs entropy has changed due to the fact that the $w$ field takes different values on the strips $(R, \hat{S}, L)$, leading to the new value 


$$
\begin{aligned}
S_{m}^{(G)} & =k_{B} \gamma \varrho a b\left\{g \ln \left[w_{m-1}\left(1+\tau q_{m}\right)\right]+(1-2 g) \ln \left[w_{m}\left(1+\tau q_{m}\right)\right]+g \ln \left[w_{m-1}\left(1+\tau q_{m}\right)\right]\right\} \\
& =k_{B} \gamma \varrho a b\left\{\ln \left[w_{m}\left(1+\tau q_{m}\right)\right]+g \ln \frac{w_{m-1}}{w_{m}}-g \ln \frac{w_{m}}{w_{m+1}}\right\} .
\end{aligned}
$$

On the other hand, the coarse-grained entropy after one time step is

$$
S_{m}^{\prime}=-k_{B} a b \gamma \varrho \ln w_{m}^{\prime}
$$

\section{B. Entropy balance}

The coarse-grained entropy only depends on the average kinetic-energy density $w_{m}$, and is therefore considered as the multibaker analogue of the thermodynamic entropy. Its temporal change can be decomposed as

$$
\begin{aligned}
\frac{\Delta S_{m}}{a b \tau} & \equiv \frac{S_{m}^{\prime}-S_{m}}{a b \tau} \\
& =\frac{\left(S_{m}{ }^{\prime}-S_{m}^{(G)^{\prime}}\right)-\left(S_{m}-S_{m}^{(G)}\right)}{a b \tau}+\frac{S_{m}^{(G)^{\prime}}-S_{m}^{(G)}}{a b \tau},
\end{aligned}
$$

where information-theoretic arguments [20,21,25, 14] lead one to identify

$$
\frac{\Delta_{i} S_{m}}{a b \tau} \equiv \frac{\left(S_{m}{ }^{\prime}-S_{m}^{(G)^{\prime}}\right)-\left(S_{m}-S_{m}^{(G)}\right)}{a b \tau}
$$

with the rate of entropy production, and

$$
\frac{\Delta_{e} S_{m}}{a b \tau} \equiv \frac{S_{m}^{(G)^{\prime}}-S_{m}^{(G)}}{a b \tau}
$$

with the entropy flux. Note that the second term of the numerator of $\Delta_{i} S_{m}$ vanishes due to the initial condition of uniform fields in the cells.

Inserting the values for $S_{m}^{\prime}$ and $S_{m}^{(G)^{\prime}}$ into the definition (32a) yields

$$
\begin{aligned}
\frac{\Delta_{i} S_{m}}{a \tau} & =\frac{k_{B} \gamma \rho}{M \tau}\left[\ln \left(\frac{w_{m}^{\prime}}{w_{m}}\left(1+\tau q_{m}\right)^{-1}\right)\right. \\
& \left.-g \ln \frac{w_{m-1}}{w_{m}}-g \ln \frac{w_{m+1}}{w_{m}}\right] .
\end{aligned}
$$

Remarkably, this expression for the entropy production does not depend on the source term $Q_{m}$, but only on the values of the coarse-grained field $w$ in cell $m$ and its neighbors. The shear flow enters only indirectly through the update $w_{m}^{\prime}$ of $w_{m}$.

Similarly to the other balance equations, the entropy flux can be written as

$$
\frac{\Delta_{e} S_{m}}{a \tau}=-\frac{j_{m+1}^{(s)}-j_{m}^{(s)}}{a}+\Phi_{m}^{(t h)},
$$

where the discrete entropy current $j_{m}^{(s)}$ takes the form

$$
j_{m}^{(s)}=-k_{B} \frac{a g}{\tau} \gamma \frac{\rho}{M} \ln \frac{w_{m+1}}{w_{m}},
$$

and

$$
\Phi_{m}^{(t h)}=k_{B} \gamma \frac{\rho}{M} q_{m}
$$

describes the direct flux into the thermostat. Such a flux is encountered whenever there is a non-vanishing source term $q_{m}$. In view of Eq. (23) this finding further supports the interpretation of $q_{m}$ and $Q_{m}$ given at the end of Sect. IIID.

\section{THE MACROSCOPIC LIMIT}

In this section we evaluate the expressions of the different quantities considered in Sects. III and IV, and identify conditions for consistency with the thermodynamic results described in Sect. II].

\section{A. Definition of the limit}

The macroscopic limit implies that $L \gg a$ (i.e., $N \gg$ 1 ), and $\tau$ is much smaller than typical macroscopic time scales. Formally it can be defined as

$$
a, \tau \rightarrow 0
$$

such that the spatial coordinate

$$
x \equiv a m
$$

is finite. As mentioned earlier, the field $w$ is assumed to go over into the local temperature $T(x)$ in the macroscopic limit, i.e.,

$$
w_{m} \rightarrow C k_{B} T(x)
$$

where $C$ is a dimensionless constant. 


\section{B. The transport equations}

The macroscopic form of the velocity current (16) becomes

$$
j_{m}^{(v)}=-\frac{a^{2} g}{\tau} \partial_{x} v
$$

In order to achieve a meaningful thermodynamic result the ratio $a^{2} g / \tau$ has to be finite in the limit. Indeed, comparison with Eq. (8a) shows that

$$
\nu \equiv \frac{a^{2} g}{\tau}
$$

is the kinematic viscosity.

Equations (39) and (40) imply that Eq. (14) is the discrete form of the Navier-Stokes equation (8a) for the laminar flow. Moreover, with this choice for $g$ one obtains for the heat current (25)

$$
j^{(w)}=-\frac{\rho}{M} \nu C k_{B} \partial_{x} T .
$$

Hence, Fick's law of heat conduction is recovered with the heat conduction coefficient

$$
\lambda=\frac{\rho}{M} \nu C k_{B}
$$

Due to Eq. (42), the macroscopic limit of Eq. (21) reduces to Eq. (8b) for the temperature evolution:

$$
\partial_{t} T=\frac{\lambda}{C k_{B} \rho / M} \partial_{x}^{2} T+\frac{\nu M}{C k_{B}}\left(\partial_{x} v\right)^{2}+q T .
$$

Comparing the coefficients in this equation with the ones in Eq. (8b), one obtains

$$
C k_{B}=c_{V} M
$$

The proportionality constant $C$ introduced in Eq. (38) corresponds thus to the specific heat at constant volume (measured in units of $k_{B}$ ).

\section{The entropy balance}

In the macroscopic limit the rate of irreversible entropy production (33) becomes

$$
\frac{\Delta_{i} S_{m}}{a \tau} \rightarrow \sigma^{(i r r)}=k_{B} \gamma \nu \frac{\rho}{M}\left(\frac{\partial_{x} T}{T}\right)^{2}+\frac{\nu \rho}{T}\left(\partial_{x} v\right)^{2} .
$$

It fully agrees with the thermodynamic form of the entropy production (9a) when the coefficient $k_{B} \gamma \nu \rho / M$ in front of the first term is the heat conductivity. In view of (42) we thus conclude, that in our model $\gamma=C$, i.e., the exponent $\gamma$ appearing in the definition (26) of the entropy is proportional to the specific heat. Hence, the final form of the heat conductivity $\lambda$ can be settled to

$$
\lambda=\frac{\rho \gamma k_{B} \nu}{M}=\rho c_{V} \nu
$$

Working out the expression (35a) of the entropy current $j_{m}^{(s)}$, one finds in the macroscopic limit

$$
j^{(s)}(x)=-k_{B} \gamma \nu \frac{\rho}{M} \frac{\partial_{x} T}{T}=-\lambda \frac{\partial_{x} T}{T} .
$$

In view of 46 ) this relation also fully agrees with its thermodynamic counterpart $9 \mathrm{~b}$.

\section{BOUNDARY CONDITIONS}

In this section we demonstrate how boundary conditions for the shear flow can be implemented in the multibaker dynamics.

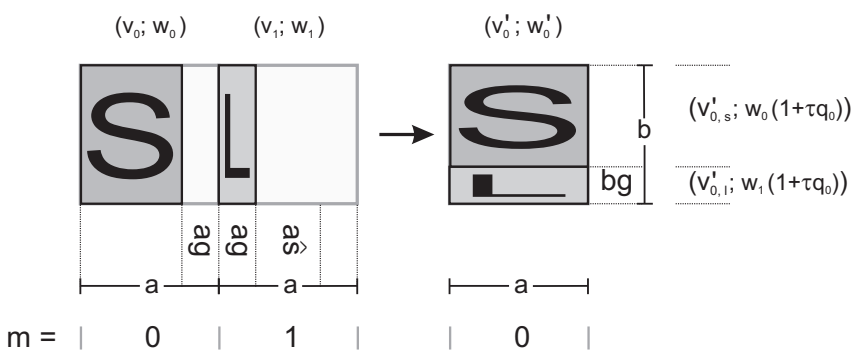

FIG. 4. Implementation of the boundary condition in cell 0 . The indication of the width of strips, values of the fields on the strips, and action of the map follows the rules spelled out in Fig. 3.

\section{A. Boundary conditions for the velocity field}

The multibaker dynamics for cell 0 is shown in Fig. 4. (An analogous prescription holds in cell $N+1$.) The action of the strip mapped from cell 0 to 1 fully agrees with the one of the corresponding strips in the bulk such that the dynamics of cell 1 agrees with the bulk dynamics of Fig. 3b. The important difference about the dynamics at the boundary is that

(i) there is no column leaving cell 0 to the left, and

(ii) the momentum of the particles entering cell 0 is not preserved.

Condition (i) reflects that no particles can penetrate the walls of the system, and (ii) reflects that there is a force exerted by the boundary on the fluid. In strip $L$ entering cell 0 (and analogously strip $R$ entering cell $N+1$ ) the update of the velocity consequently contains the contribution of the external force $f_{0}\left(f_{N+1}\right)$, leading to

$$
\begin{aligned}
v_{0, l}^{\prime} & =v_{1}+\frac{f_{0} \tau}{g \rho} \\
v_{N+1, r}^{\prime} & =v_{N}+\frac{f_{N+1} \tau}{g \rho} .
\end{aligned}
$$


The update of the coarse-grained velocity of the leftmost cell is

$$
v_{0}^{\prime}=v_{0}+g\left(v_{1}-v_{0}\right)+\frac{f_{0} \tau}{\varrho},
$$

and an analogous relation holds for cell $N+1$. A shear flow with a prescribed shear rate is enforced by adjusting the forces in such a way that $v_{0}$ and $v_{N+1}$ take the respective values $v_{L}$ and $v_{R}$ of the velocities at the walls at any time.

In view of the time evolution of $v_{m}$, in a steady state [Eq. (14) with $v_{m}^{\prime}=v_{m}$ ], the asymptotic velocity profile becomes linear

$$
v_{m}=v_{L}+\frac{m}{N+1}\left(v_{R}-v_{L}\right) \equiv v_{L}+\frac{m}{N+1} \Delta v
$$

irrespective of the temperature distribution in the system.

\section{B. Boundary conditions for the kinetic-energy density}

The modification of the dynamics of the velocities at the boundary also implies changes of the thermostat source terms. Since the velocity in cell 0 is uniform by definition, the update of $w_{0}$ can be found from Eq. 18) where the terms containing $w_{m, l}$ and $v_{m, l}$ are not present since no particles enter cell 0 from the left. Consequently,

$$
w_{0}^{\prime}=\left[w_{0}+g\left(w_{1}-w_{0}\right)\right]\left(1+\tau q_{0}\right) .
$$

This leads to the discrete temporal change

$$
\frac{w_{0}^{\prime}-w_{0}}{\tau}=\frac{q_{0}}{1+\tau q_{0}} w_{0}^{\prime}+\frac{1}{a} \frac{a^{2} g}{\tau} \frac{w_{1}-w_{0}}{a}
$$

instead of Eq. (22). For a steady state $w_{0}^{\prime}-w_{0}$ vanishes, and the equation implies [cf. Eq. (25)]

$$
a q_{0}=-\frac{a^{2} g}{\tau} \frac{\left(w_{1}-w_{0}\right) / a}{w_{0}+g\left(w_{1}-w_{0}\right)}=-\frac{j_{1}^{(w)}}{\varrho\left[w_{0}+g\left(w_{1}-w_{0}\right)\right]}
$$

The right-hand side of this expression is finite in the macroscopic limit, where

$$
a q_{0} \rightarrow-\left.\nu \frac{\partial_{x} T}{T}\right|_{x=0}=-\frac{\nu j^{(T)}(0)}{\lambda T(0)}
$$

Since, however, $a \rightarrow 0$ in the macroscopic limit, the thermostat heat source $q_{0}$ cannot be interpreted as a density when there is a finite heat current $j^{(w)}(0)$. Its integral over the cell, $a q_{0}$, stays however finite. As a consequence, the heat current has to vanish in a steady state when $q_{0}=q_{N+1}=0$.

\section{DIFFERENT MACROSCOPIC FLOWS}

We have seen that in the macroscopic limit the local momentum, energy and entropy balances for the multibaker map coincide with their thermodynamic forms. This result was achieved by inspecting the local time evolution of the densities without referring to particular boundary conditions, and it is not restricted to steady states. In order to underline these features, we discuss the global transport pictures for three different settings of transport. We emphasize, that the considerations immediately generalize to arbitrary time-dependent states although the particular calculations are carried out for stationary coarse-grained velocity fields.

\section{A. Isolated systems}

In thermodynamics sheared systems are often considered to be thermally isolated. In that case $q_{m}$ identically vanishes for all cells $m=0, \cdots, N+1$. Eq. (51) implies for the current 25)

$$
j_{1}^{(w)} \equiv-\frac{a^{2} g}{\tau} \frac{\rho}{M} \frac{w_{1}-w_{0}}{a}=-\frac{a \rho}{M} \frac{w_{0}^{\prime}-w_{0}}{\tau} .
$$

Assuming that the time derivative of $w_{0}$ is finite in the macroscopic limit, the current $j_{1}^{(w)} \rightarrow$ $-a(\rho / M) C k_{B} \partial_{t} T(0)$ approaches zero as $a \rightarrow 0$. An analogous arguments applies also to $j_{N+1}^{(w)}$.

Thus, for the asymptotic state, the boundary conditions imply that $w_{0}$ and $w_{N+1}$ practically coincide with $w_{1}$ and $w_{N}$, respectively. Moreover, the viscous heating term $Q_{m}$ in the bulk only depends on the square of $v_{m+1}-v_{m}$. The heating is therefore spatially uniform in the long time limit. With this input, one immediately verifies that $w_{m}$ approaches a spatially uniform value $w^{*}$. However, in view of the heat source $Q_{m}$ in (23), it constantly grows in time,

$$
\frac{w^{*^{\prime}}-w^{*}}{\tau}=\nu M\left(\frac{\Delta v}{a(N+1)}\right)^{2}
$$

This temporal evolution of $w$ reflects the rise of temperature due to the entropy production in the system. After all,

$$
\frac{\Delta_{i} S_{m}}{a \tau} \equiv \frac{k_{B} \gamma \rho}{\tau M} \ln \frac{w^{*^{\prime}}}{w^{*}}=\frac{k_{B} \gamma \rho}{w^{*}} \nu\left(\frac{\Delta v}{a(N+1)}\right)^{2}+\mathcal{O}(\tau)
$$

which in the macroscopic limit reduces to the thermodynamically expected value

$$
\sigma^{(i r r)}(t)=\frac{\rho \nu}{T^{*}(t)}\left(\frac{\Delta v}{L}\right)^{2}
$$

where $T^{*}(t)=$ const $+\left(\nu / c_{V}\right)(\Delta v / L)^{2} t$ is the spatially uniform, but temporally increasing asymptotic temperature distribution. Note that the flux $\Phi^{(t h)}$ and the entropy current vanishes in this setting, implying an ever increasing entropy. 


\section{B. Systems subjected to an ideal (bulk) thermostat}

In a system subjected to an ideal thermostat the viscous heating is instantaneously released into a heat bath. Accordingly, $Q_{m}$ vanishes for all cells $m=1, \cdots, N$ such that $q_{m}$ can be determined from Eq. (23).

Moreover, according to the results obtained in Sect. VIB we are again dealing with a system with vanishing heat currents through its boundaries, i.e., for the asymptotic state the boundary conditions imply $w_{0}=w_{1}$ and $w_{N}=w_{N+1}$, and there is no source term $Q$ for the heat. Consequently, $w_{m}$ approaches a spatially uniform state $w^{*}$ that is stationary in this case. Entropy production arises due to the nontrivial form of $q_{m}$ [cf. Eq. 23] with $\left.Q_{m}=0\right]$

$$
q_{m} \equiv q^{*}=-\frac{\nu M\left(\frac{\Delta v}{a(N+1)}\right)^{2}}{w^{*}+\nu M \tau\left(\frac{\Delta v}{a(N+1)}\right)^{2}}
$$

in the bulk [cf. the steady-state velocity profile (50)]. Hence, the irreversible entropy change is

$$
\begin{aligned}
\frac{\Delta_{i} S_{m}}{a \tau} & \equiv-\frac{k_{B} \gamma b \varrho}{\tau} \ln \left(1+\tau q^{*}\right) \\
& =\frac{k_{B} \gamma \rho}{M w^{*}} \nu \frac{\Delta v^{2}}{a^{2}(N+1)^{2}}+\mathcal{O}(\tau),
\end{aligned}
$$

and in the macroscopic limit it reduces to the thermodynamically expected value

$$
\sigma_{m}^{(i r r)}=\frac{\rho \nu}{T^{*}}\left(\frac{\Delta v}{L}\right)^{2}
$$

where $T^{*}$ is the steady-state temperature. Also in this case there is no entropy current in the steady state. However, at every location in the system there is a nonvanishing entropy flux into the thermostat

$$
\Phi^{(t h)}=k_{B} \gamma b \varrho q_{m} \rightarrow-\frac{\rho \nu}{T^{*}}\left(\frac{\Delta v}{L}\right)^{2},
$$

which exactly compensates $\sigma^{(i r r)}$ such that the entropy indeed is stationarity.

\section{Thermostating at the walls}

Finally, we discuss a steady state in a hydrodynamic bulk system that generates heat flux into the walls due to the prescribed temperatures at the boundaries. There are no thermostat heat sources in the bulk, i.e., $q_{m}=0$ for $m=1, \cdots, N$. On the other hand, there are source terms $q_{0}$ and $q_{N+1}=q_{0}$ acting in the two outermost cells that fix the values of $w_{0}$ and $w_{N+1}$ (i.e., the temperature) to the same constant value $w_{0}$. To this end the sources counterbalance a macroscopic heat current releasing the viscous heat into the bath at the boundaries [cf. Eq. (52)]. In contrast to the previous cases, the discrete heat current does not vanish at the boundaries. In view of Eqs. (25) and (51) the heat current through the left boundary of the steady state system is

$$
\frac{j_{1}^{(w)}}{\rho} \equiv-\nu \frac{w_{1}-w_{0}}{a}=\frac{a q_{0}}{1+\tau q_{0}} w_{0} .
$$

Since the dynamics is symmetrical and $w_{N+1}=w_{0}$, the current at the right boundary takes the same value up to a change of the sign

$$
j_{N+1}^{(w)}=-j_{1}^{(w)}
$$

In a steady state the cells in the bulk consequently fulfill

$$
0=\rho \frac{w_{m}^{\prime}-w_{m}}{\tau}=\rho \nu \frac{\Delta v^{2}}{a^{2}(N+1)^{2}}-\frac{j_{m+1}^{(w)}-j_{m}^{(w)}}{a}
$$

leading to a linear profile of the heat current,

$$
j_{m}^{(w)}=a\left(m-1-\frac{N}{2}\right) \rho \nu\left(\frac{\Delta v}{a(N+1)}\right)^{2} .
$$

In view of the definition Eq. (25) of $j_{m}^{(w)}$, this implies a parabolic profile of $w$ in the steady state,

$w_{m}^{*}=w_{0}-\frac{a m}{2} a[m-(N+1)] M\left(\frac{\Delta v}{a(N+1)}\right)^{2}$,

as expected for the temperature profile in a shear flow subjected to identical temperatures at the two ends.

The entropy production in the bulk is related to the spatial variation of the steady-state distribution $w_{m}^{*}$ [cf. Eq. (33)],

$$
\begin{aligned}
\sigma_{m}^{(i r r)} & \equiv-\frac{k_{B} \gamma \rho}{\tau M}\left[g \ln \frac{w_{m-1}^{*}}{w_{m}^{*}}+g \ln \frac{w_{m+1}^{*}}{w_{m}^{*}}\right] \\
& =\frac{k_{B} \gamma}{M}\left[\frac{1}{w_{m}^{*}} \frac{j_{m+1}^{(w)}-j_{m}^{(w)}}{a}+\frac{j_{m+1}^{(w)^{2}}+j_{m}^{(w)^{2}}}{2 \rho \nu w_{m}^{* 2}}\right],
\end{aligned}
$$

where the second equation was obtained by expanding the logarithms to second order in the differences $w_{m+1}^{*}-w_{m}^{*}$, and rearranging terms. Using Eqs. (24), (23) and (59), the first term can be related to the applied shear rate, while the second one represents the contribution to the entropy production arising from the heat flow. Indeed, in the macroscopic limit one recovers in this case both contributions to Eq. (45). (For small shear rates the contribution from the temperature change is, however, negligible). Correspondingly, there is a finite entropy current $j^{(s)}$ at every point in the system, but only at the boundaries there is a flux into the thermostat. According to Eq. (53) the macroscopic limit of the full entropy flux through the boundaries becomes 


$$
a \Phi^{(t h)}=\left.k_{B} \gamma b \varrho a q_{0} \rightarrow k_{B} \gamma \nu \frac{\rho}{M} \frac{\partial_{x} T}{T}\right|_{x=0} .
$$

Since the stationary temperature profile is obtained from (60) in the form of $T(x)=T(0)-[x(x-$ $\left.L)\left(M / 2 C k_{B}\right)\right](\Delta v / L)^{2}$, the derivative at the origin is $\left.\partial_{x} T\right|_{x=0}=M L(\Delta v / L)^{2} /\left(2 C k_{B}\right)$. The contribution at the right boundary is the same. Therefore, we find for the integrated entropy flux

$$
\Phi^{(t h, t o t)}=-\frac{\rho \nu}{T_{0}} L\left(\frac{\Delta v}{L}\right)^{2},
$$

where we used $\gamma=C$. For weak shear this is essentially the same as the integral of the constant flux (57) over the chain. Thus, we conclude that for sufficiently small heat currents thermostating in the bulk and in the boundaries can lead to the same global behavior.

The work per unit time done by the external force densities $f_{0}$ and $f_{N+1}$ can be evaluated using their form Eq. (48) and the value of the constant velocity gradient

$$
a\left(f_{0} v_{L}+f_{N+1} v_{R}\right)=\nu \rho \frac{(\Delta v)^{2}}{L} .
$$

This expression is exactly $-T_{0} \Phi^{(t h, t o t)}$, such that in a steady state the work done by the external forces equals the total heat flux into the thermostat.

\section{DISCUSSION}

We have enlarged the family of multibaker maps by a model for momentum, energy and entropy transport in viscous hydrodynamic flows. Although the macroscopic problem is of strongly dissipative nature, the proposed multibaker dynamics is area preserving (Hamiltonian). This is to be contrasted with previous multibaker models of electric transport [20,21] and thermoelectric cross effects [25,26] where the inclusion of a reversible dissipation mechanism was necessary in order to simulate the effect of thermostating on the particle dynamics. The form in which thermostating appears in the present model is via a heat-source term in the microscopic energy dynamics (which was already present in the model of cross effects [25]). This term, however, does not give rise to phasespace contraction.

The model has the following basic features:

(a) The time evolution of the system can be interpreted as that of weakly interacting particles. The resulting "multi-baker" gas obeys the classical ideal-gas equation of state. The particle and heat diffusion coefficient, and the kinematic viscosity are proportional to each other, as in the kinetic theory for classical ideal gases [27].

(b) The distribution of a macroscopic velocity does not enter the entropy explicitly. Rather the shear rate appears in the entropy balance via its influence on the temperature dynamics only. (c) The connection to a thermodynamic description of transport is achieved by considering fields, which are coarse grained in regions of small spatial extension. Their properties are to be contrasted to those of fields characterizing the microscopic evolution.

(d) Comparing these two levels of description allows us to identify all contributions to the local entropy balance, in full consistency with thermodynamics. They apply to both stationary and transient states.

(e) A source term is implemented in the evolution equation of the microscopic kinetic energy. It provides the possibility to implement local irreversible cooling of the system, i.e., to extract heat such that states which are permanently warmed up by viscous heating can become stationary. In traditional thermodynamics these terms are only present at the boundaries, and they vanish in the bulk.

(f) It is indicated how the velocity and the kineticenergy dynamics can be implemented at the walls in order to achieve correspondence with different macroscopic boundary conditions.

(g) When source terms are present in the bulk, the local entropy balance of nonequilibrium thermodynamics is generalized by introducing at every location an instantaneous flux of entropy (i.e., of heat) into a thermostat (the entropy flux is then no longer the divergence of the entropy current). The dynamics is in that case reminiscent of numerical algorithms based on Gaussian thermostats. (h) The global entropy balance was worked out, in order to demonstrate that the total heat flux into a thermostat is independent to a large extend of whether thermostating is applied in the bulk or at the walls.

The major interest of the present model lies in the light it sheds on the origin of viscous heating in deterministic models of transport. It was pointed out how fractal structures emerge in multibaker models for a variety of physical settings of shear flow. In all cases the structures arise from the mixing of regions with different local temperatures and flow velocities whose differences are exponentially proliferating to smaller and smaller scales for a nonequilibrium system. One can explicitly follow this redistribution of the kinetic energy, until it reaches the scale of the domains used to define local thermodynamic averages. Motion at that scale has to be considered as contributing to the non-directional motion, hence leading to viscous heating. It is only due to this coarse-graining mechanism that the macroscopic shear rate appears in the expression of the irreversible entropy production.

\section{ACKNOWLEDGMENTS}

We would like to thank Bob Dorfman, Christan Gruber, and Gary Morris for illuminating discussions. Support from the Hungarian Science Foundation (OTKA Grand No. 032423) is acknowledged. 
[1] S.R. de Groot and P. Mazur, Non-Equilibrium Thermodynamics (Elsevier, Amsterdam, 1962) (reprinted: Dover, New-York, 1984).

[2] R. Balescu, Statistical Dynamics (Imperial College Press, 1997).

[3] D.J. Evans and G.P. Morriss, Statistical Mechanics of Nonequilibrium Liquids (Academic Press, London, 1990); W.G. Hoover, Computational Statistical Mechanics (Elsevier, Amsterdam, 1991).

[4] J.R. Dorfman, An Introduction to Chaos in NonEquilibrium Statistical Mechanics (Cambridge Univ. Press, Cambridge, 1999).

[5] CHAOS 8, No.2 (1998), Focus issue on Chaos and Irreversibility.

[6] D.J. Evans, E.G.D. Cohen, and G.P. Morriss, Phys. Rev. Lett. 71, 2401 (1993); erratum, 71, 3616 (1993).

[7] N.I. Chernov and J.L. Lebowitz, Phys. Rev. Lett. 75, 2831 (1995); J. Stat. Phys. 86, 953 (1996).

[8] Ch. Dellago and H. Posch, J. Stat. Phys. 88, 825 (1997); Ch. Dellago, W.G. Hoover, and H. Posch, Phys. Rev. E 57, 4969 (1998); G. Ayton, O.G. Jepps, and D.J. Evans, Molecular Physics 96, 915 (1999).

[9] Ch. Wagner, R. Klages and G. Nicolis, Phys. Rev. E 60, 1404 (2000).

[10] L.A. Bunimovich and H. Spohn, Comm. Math. Phys. 176, 661 (1996).

[11] G. Morriss and L. Rondoni, Physica A 233, 767 (1996).
[12] P. Gaspard, Chaos, Scattering and Statistical Mechanics (Cambridge Univ. Press, Cambridge, 1998).

[13] T. Tél and J. Vollmer, in D. Szász, ed.: Hard Ball systems and the Lorentz Gas (Springer, Berlin, 2000).

[14] J. Vollmer, Chaos, Spatial Extension and Non-Equilibrium Thermodynamics, http://www.mpip-mainz.mpg.de/ vollmer/habil.pdf.

[15] L. Rondoni and E.G.D. Cohen, Nonlinearity 13, 1905 (2000).

[16] P. Gaspard, J. Stat. Phys. 68, 673 (1992).

[17] S. Tasaki and P. Gaspard, J. Stat. Phys. 81, 935 (1995).

[18] P. Gaspard, Physica A 240, 54 (1997); J. Stat. Phys. 88, 1215 (1997).

[19] T. Tél, J. Vollmer, and W. Breymann, Europhys. Lett. 35, 659 (1996).

[20] J. Vollmer, T. Tél, and W. Breymann, Phys. Rev. Lett. 79, 2759 (1997).

[21] J. Vollmer, T. Tél, and W. Breymann, Phys. Rev. E 58, 1672 (1998); W. Breymann, T. Tél, and J. Vollmer, CHAOS 8, 396 (1998).

[22] T. Gilbert, C.D. Ferguson, and J.R. Dorfman, Phys. Rev. E 59, 364 (1999).

[23] P. Gaspard and R. Klages, CHAOS 8, 409 (1998).

[24] S. Tasaki and P. Gaspard, Theoretical Chemistry Accounts 102, 385 (1999).

[25] L. Mátyás, T. Tél, and J. Vollmer, Phys. Rev. E 61, R3295 (2000); 62, 349 (2000).

[26] J. Vollmer, T. Tél, and L. Mátyás, J. Stat. Phys. 101, 79 (2000).

[27] L.E. Reichl Statistical Physics, (John Wiley \& Sons, 1998). 